\title{
Prescribing Patterns of Antihypertensives for Treatment-Naïve Patients in South Korea: From Korean NHISS Claim Data
}

\author{
Sang Hyuck Kim, ${ }^{1}$ Dong Wook Shin $\mathbb{D}^{\mathrm{D}},{ }^{2}$ Shinhye Kim, ${ }^{3}$ Kyungdo Han, ${ }^{4}$ Sang-hyun Park, ${ }^{4}$ \\ Yul-Hee Kim, ${ }^{5}$ Shin-Ae Jeon, ${ }^{5}$ and Yong-Chol Kwon ${ }^{5}$ \\ ${ }^{1}$ Department of Family Medicine, Bumin Hospital, Seoul, Republic of Korea \\ ${ }^{2}$ Department of Family Medicine, Samsung Medical Center, Sungkyunkwan University School of Medicine, \\ Seoul, Republic of Korea \\ ${ }^{3}$ Department of Family Medicine, Gangnam Severance Hospital, Seoul, Republic of Korea \\ ${ }^{4}$ Department of Biostatistics, College of Medicine, Catholic University, Republic of Korea \\ ${ }^{5}$ Medical Affairs, Pfizer Essential Health Business Unit, Pfizer Pharmaceuticals Korea Limited, Seoul, Republic of Korea
}

Correspondence should be addressed to Dong Wook Shin; dwshin.md@gmail.com

Received 22 January 2019; Revised 10 July 2019; Accepted 30 July 2019; Published 25 August 2019

Academic Editor: Tomohiro Katsuya

Copyright (c) 2019 Sang Hyuck Kim et al. This is an open access article distributed under the Creative Commons Attribution License, which permits unrestricted use, distribution, and reproduction in any medium, provided the original work is properly cited.

\begin{abstract}
Background. Several factors influence the choice of antihypertensive drugs. To facilitate the rational use of drugs it is important to assess their prescription patterns over time. This study aims to evaluate doctors' prescribing patterns of antihypertensive drugs for drug-naïve patients in South Korea. Methods. The claims data of the Korean National Health Insurance Research Database from 1 January 2011 to 31 December 2015 were analyzed. The data virtually cover the entire South Korean population. Antihypertensive drugs were further subdivided into angiotensin receptor blockers (ARBs), angiotensin-converting enzyme inhibitors (ACEis), calcium channel blockers (CCBs), beta-blockers (BBs), and thiazide diuretics. The prescription pattern of antihypertensive drugs and associated factors were assessed according to the patients' characteristics, including associated comorbidities. Results. A total of 2,919,162 subjects had started taking antihypertension medications during the study period. ARB was the most frequently prescribed drug (51.6\%) followed by CCB (45.0\%), BB (18.5\%), diuretics (17.0\%), and ACEi (11.7\%). Most patients were prescribed with monotherapy (66.7\%) rather than combination therapy $(33.3 \%)$, and CCB was the most frequently prescribed monotherapy drug (25.7\%). For combination therapy, ARB + CCB was the most frequently prescribed combination, and the prescription frequency was found to be increasing. In patients prescribed with combination therapy, most had been prescribed single-pill fixeddose combination. Conclusion. We identified the physicians' prescription patterns of antihypertensive drugs for treatment-naïve patients. The findings of this study can lead to a rational, evidence-based, and cost-effective improvement of prescription patterns in newly diagnosed hypertensive patients.
\end{abstract}

\section{Introduction}

Hypertension is a common chronic condition which is associated with various complications including cardiovascular disease and chronic kidney disease. It remains that hypertension is one of the most important preventable conditions to reduce cardiovascular mortality [1]. Despite this, the high and increasing prevalence of hypertension still contributes to considerable socioeconomic burden globally [2-4].
In order to choose the appropriate antihypertensive drug among various available classes, several factors should be considered, such as age and underlying comorbid conditions [5-7]. Also, the type of antihypertensive drugs can affect prescribing patterns as well as drug compliance [8-11]. Therefore, it is necessary to assess prescription patterns to determine whether current prescription is reasonable, evidence-based, and cost-effective [11]. Although there have been several studies on prescription patterns of antihypertensives in other countries, they are not relevant in the 
Korean context since prescription patterns are influenced by time and region $[9,10,12-14]$. Furthermore, previously conducted studies in Korea are either too old or not representative (single hospital-based study) $[15,16]$.

This study aimed at evaluating prescribing patterns of antihypertensive drugs in patients who were prescribed antihypertensive drugs for the first time.

\section{Materials and Methods}

2.1. Data Sources. The claims data of the Korean National Health Insurance Research Database (NHIRD) were analyzed. NHIRD contains qualification data, medical services claim data, and pharmacy claim data. As such, the claims data include patient information such as age, sex, household income, residential regions, as well as diagnosis information (by the International Classification of Diseases, 10th Revision; ICD-10) and specific information of diagnostic tests, procedures, and prescriptions.

In general, the Korean National Health Insurance Service (NHIS) is the sole insurer providing a mandatory universal health insurance which virtually covers the entire Korean population (about $97 \%$ of total population) and a medical aid program to those in the lowest income bracket who are covered by government funding. We used the nation-wide claims data which covered the South Korean population over a 5-year period, from 1 January 2011 to 31 December 2015. Detailed insights into the advantages of this data are described elsewhere $[17,18]$.

2.2. Study Population. From the whole Korean population ( $N=\sim 50$ million), patients who were newly diagnosed with hypertension (ICD-10: I10, I11, I12, I13, or I15) and prescribed with antihypertensive agents were included in this study. We further confirmed that the study subjects did not have any previous record of antihypertensive medication during the prior 12 months.

\subsection{Variables}

2.3.1. Antihypertensive Agents. Antihypertensive agents were classified into 5 major categories, including angiotensin receptor blockers $(\mathrm{ARB})$, angiotensin-converting enzyme inhibitors (ACEi), calcium channel blockers (CCB), betablockers (BB), and thiazide diuretics (including indapamide and chlorthalidone), or other antihypertensive drugs.

Initial prescription was divided into single drug and combination. Single drug was defined as the prescription of 1 class of antihypertensive at first prescription. Combination was defined as prescription of 2 or more classes of antihypertensives at first prescription and was further categorized by single-pill fixed-dose drug and free combination (multiple-pill combinations). Commonly used combinations available commercially are specifically listed: $\mathrm{ARB}+\mathrm{CCB}, \mathrm{ARB}+$ thiazide, and $\mathrm{ARB}+\mathrm{CCB}+$ thiazide.

2.3.2. Comorbidities. Comorbidities were defined by ICD 10 codes: diabetes (ICD 10: E11-14), dyslipidemia (E78), congestive heart failure (I50), coronary heart disease(I2025), stroke (I60 to I64, I67, and I69), chronic kidney disease (N18), and cancer (CX) during the previous 1 year prior to index date (first prescription of antihypertensive medication). The Charlson comorbidity index was also assessed in the same manner [16].

2.4. Statistical Analyses. The baseline characteristics of hypertensive patients who were newly prescribed with antihypertensive medication were described using frequencies and percentages or as means with standard deviation.

Descriptive statistics were calculated for patterns of first prescriptions of antihypertensive medications. The proportion of each antihypertensive class was calculated according to patient characteristics (age, sex, income level, and place of residence), medical characteristics (the Charlson comorbidity index and each comorbidity as defined above), provider characteristics (level of hospital; general hospital vs. hospital vs. clinic), and year of first prescription.

Factors associated with choice of first prescription for hypertension were investigated with a series of multivariate logistic regression analyses including all of the aforementioned characteristics. Outcomes were (1) combination vs. single, (2) choice of each drug class among single drug user (exclusive to each other), (3) choice of each drug class among all patients (not exclusive to each other), and (4) fixed-dose combination vs. free combination for selected combinations. Also, the trends of combination therapy according to the age group, income level, and Charlson's comorbidity index were assessed by calculating the " $p$ for trend."

All analyses were performed using the SAS statistical software (ver. 9.3, SAS Institute., Cary, NC, USA). All tests were two-sided, and statistical significance was defined as a $p$ value $<0.05$

2.5. Ethics Statement. This study was reviewed and approved by the Institutional Review Board of the Samsung Medical Center (IRB No. SMC 2007-07-130). The requirement for informed consent was waived because this study is based on routinely collected administrative or claims data.

\section{Results}

3.1. Baseline Characteristics. A total of 2,919,162 subjects had started taking antihypertension medications during the study period. $56.0 \%$ of these were male patients. The mean age of the study population was $53.0( \pm 14.5)$ years. Over half of the study population resided in metropolitan areas. About $67.9 \%$ of subjects had 1 or more comorbidity as per the Charlson comorbidity index. Subjects had various hypertension-related comorbid conditions such as dyslipidemia (27.6\%), coronary heart disease $(13.8 \%)$, diabetes $(12.7 \%)$, stroke, $(4.6 \%)$, congestive heart failure $(4.1 \%)$, and chronic kidney disease $(0.93 \%)$. The annual incidence rate per year was $1.24 \%$ in $2011,1.20 \%$ in $2012,1.13 \%$ in $2013,1.05 \%$ in 2014 , and $1.12 \%$ in 2015 , respectively (Table 1 ). 
TABLE 1: Baseline characteristics of newly diagnosed hypertension patients (total $n=2,919,162$ )

\begin{tabular}{|c|c|c|}
\hline & $n$ & $\%$ \\
\hline \multicolumn{3}{|l|}{ Age } \\
\hline$<20$ & 45,222 & 1.55 \\
\hline $20-29$ & 95,489 & 3.27 \\
\hline $30-39$ & 303,523 & 10.40 \\
\hline $40-49$ & 713,524 & 24.44 \\
\hline $50-59$ & 866,370 & 29.68 \\
\hline $60-69$ & 508,662 & 17.42 \\
\hline $70-79$ & 294,771 & 10.10 \\
\hline$\geq 80$ & 91,601 & 3.14 \\
\hline \multicolumn{3}{|l|}{ Sex } \\
\hline Male & $1,633,768$ & 55.97 \\
\hline Female & $1,285,394$ & 44.03 \\
\hline \multicolumn{3}{|l|}{ Income } \\
\hline Medical aid & 112,217 & 3.84 \\
\hline Q1 (low) & 766,378 & 26.25 \\
\hline Q2 & 696,643 & 23.86 \\
\hline Q3 & 663,524 & 22.73 \\
\hline Q4 (high) & 680,400 & 23.31 \\
\hline \multicolumn{3}{|l|}{ Residence } \\
\hline Metropolitan & $1,741,304$ & 59.65 \\
\hline City & 833,767 & 28.56 \\
\hline Rural & 344,091 & 11.79 \\
\hline \multicolumn{3}{|l|}{ Charlson comorbidity index } \\
\hline 0 & 938,134 & 32.14 \\
\hline 1 & 791,388 & 27.11 \\
\hline 2 & 497,523 & 17.04 \\
\hline$\geq 3$ & 692,117 & 23.71 \\
\hline \multicolumn{3}{|l|}{ Provider type } \\
\hline General hospital & 924,592 & 31.67 \\
\hline Hospital & 330,928 & 11.34 \\
\hline Clinic & $1,560,700$ & 53.46 \\
\hline Others (public health centers, etc.) & 102,942 & 3.53 \\
\hline \multicolumn{3}{|l|}{ Comorbidity } \\
\hline Diabetes & 371,477 & 12.73 \\
\hline Dyslipidemia & 806,246 & 27.62 \\
\hline Congestive heart failure & 120,749 & 4.14 \\
\hline Ischemic heart failure & 401,547 & 13.76 \\
\hline Stroke & 135,569 & 4.64 \\
\hline Chronic kidney disease & 27,263 & 0.93 \\
\hline Cancer & 44,626 & 1.53 \\
\hline \multicolumn{3}{|l|}{ Year of diagnosis } \\
\hline 2011 & 627,249 & 21.49 \\
\hline 2012 & 597,912 & 20.48 \\
\hline 2013 & 578,479 & 19.82 \\
\hline 2014 & 538,468 & 18.45 \\
\hline 2015 & 577,054 & 19.77 \\
\hline
\end{tabular}

3.2. Frequency and Trend of the Prescription. Around twothirds of the study population was prescribed with monotherapy at first prescription, and one-third was prescribed with initial combination therapy (Table 2). CCB was the most frequently prescribed monotherapy drug (25.70\%), followed by ARB (24.45\%) and BB (11.88\%). The proportion of $\mathrm{ARB}$ and $\mathrm{BB}$ prescriptions increased during the study period, while CCB and ACEI decreased. The ARB + CCB was the most frequently prescribed combination (12.59\%) and was found to be increasing, while ARB + thiazide (9.79\%) was decreasing during the study period (Table 3). Among the
5 drug classes, ARB was the most frequently prescribed drug (51.61\%), followed by CCB (45.03\%), BB (18.48\%), thiazide diuretics (17.01\%), and ACEi (3.11\%). Initial drug choice differed by comorbidities, e.g., $64.74 \%$ of diabetes patients initiated with $\mathrm{ARB}$, while $54.18 \%$ of $\mathrm{CHF}$ patients were initiated with beta-blockers. In CKD patients, $59.82 \%$ received ARB and $8.34 \%$ received ACEi (Table 4).

A comparison of prescription rates between 2011 and 2015 revealed an increase of $4 \%$ in the prescription rate of $\mathrm{ARB}$ and an $8 \%$ increase in the rate of thiazide prescription (Figure 1).

3.3. Trend of Single-Pill Fixed-Dose Combination. Among combination therapy users, most patients were prescribed a single-pill fixed-dose combination (83.73\% for ARB + CCB and $96.46 \%$ for ARB + thiazide, respectively) in an increasing proportion during the study period (Table 3 ).

3.4. Trend and Factors Associated with Initial Combination Therapy. Female patients were less likely to be prescribed with combination therapy at first prescription (adjusted odds ratio: $0.764,95 \%$ CI $0.760-0.768)$. Having congestive heart failure, dyslipidemia, CHD, stroke, CKD, and cancer was slightly associated with initiation with combination therapy, while it was opposite with diabetes mellitus. Primary clinics prescribed combination antihypertensive drugs more frequently than secondary hospitals and general hospitals. Initial combination therapy decreased slightly during the study period (35.81 in year 2011 to $32.53 \%$ in year 2015). There was no significant trend by the age group or Charlson comorbidity index (Table 2).

\section{Discussion}

In this study, physicians' prescription patterns of antihypertensive drugs for treatment-naïve patients were identified using the National Health Insurance claims data. The strength of this study is its generalizability, as it uses claims data collected from the entire South Korean population and assess data over a 5-year period which allows for the identification of trends in prescription patterns.

In a previous study of prescription patterns in Korea conducted in 2009, CCB was the most frequently prescribed antihypertensive (64.4\%), followed by diuretics (44.6\%), ARB (33.3\%), BB (21.6\%), and ACEi (11.7\%) [16]. In this study, the corresponding figures were $45.0 \%, 17.0 \%, 51.6 \%$, $18.5 \%$, and $3.1 \%$, respectively. This shows that the use of ARB has significantly increased over time, while use of other antihypertensive classes decreased.

Similarly, we also identified that the use of thiazide diuretics and ACEi was decreasing and ARB use was increasing during the study period. Although CCB was the most frequently prescribed drug for monotherapy, overall $\mathrm{ARB}$ was the most frequently prescribed class of antihypertensive drugs since the prescription of ARB for combination therapy also increased.

The prescription of CCBs and ARBs increased worldwide over the past several years [11]. Especially, CCBs and 
TABle 2: Prescription pattern by the number of drug classes, $n$ (\%).

\begin{tabular}{|c|c|c|c|}
\hline & Single drug class & Combination & Combination (vs. single) \\
\hline & & & OR $(95 \% \mathrm{CI})$ \\
\hline Overall & $1,947,796(66.72)$ & $971366(33.28)$ & \\
\hline Age $($ mean $\pm S D)$ & $53.13 \pm 14.48$ & $52.84 \pm 14.52$ & \\
\hline$<20$ & $29,435(65.09)$ & $15,787(34.91)$ & $1.214(1.190-1.239)$ \\
\hline $20-29$ & $72,099(75.51)$ & $23,390(24.49)$ & $0.650(0.640-0.661)$ \\
\hline $30-39$ & $202,994(66.88)$ & $100,529(33.12)$ & $0.957(0.948-0.965)$ \\
\hline $40-49$ & $458,093(64.2)$ & $255,431(35.8)$ & $1.094(1.087-1.101)$ \\
\hline $50-59$ & $574,198(66.28)$ & $292,172(33.72)$ & 1 (ref.) \\
\hline $60-69$ & $348,811(68.57)$ & $159,851(31.43)$ & $0.900(0.894-0.907)$ \\
\hline $70-79$ & $202,102(68.56)$ & $92,669(31.44)$ & $0.913(0.905-0.922)$ \\
\hline$\geq 80$ & $60,064(65.57)$ & $31,537(34.43)$ & $1.041(1.026-1.057)$ \\
\hline \multicolumn{4}{|r|}{ ) } \\
\hline Male & $1,048,973(64.21)$ & $584,795(35.79)$ & 1 (ref.) \\
\hline Female & $898,823(69.93)$ & $386,571(30.07)$ & $0.764(0.760-0.768)$ \\
\hline \multicolumn{4}{|l|}{ Income } \\
\hline Medical aid & $75,202(67.01)$ & $37,015(32.99)$ & $1.133(1.118-1.149)$ \\
\hline Q1(Low) & $499,304(65.15)$ & $267,074(34.85)$ & $1.220(1.212-1.229)$ \\
\hline Q2 & $457,765(65.71)$ & $238,878(34.29)$ & $1.184(1.175-1.193)$ \\
\hline Q3 & $444,104(66.93)$ & $219,420(33.07)$ & $1.115(1.107-1.123)$ \\
\hline Q4(High) & $471,421(69.29)$ & $208,979(30.71)$ & 1 (ref.) \\
\hline \multicolumn{4}{|l|}{ Residence } \\
\hline Metropolitan & $1,172,041(67.31)$ & $569,263(32.69)$ & 1 (ref.) \\
\hline City & $549,150(65.86)$ & $284,617(34.14)$ & $1.068(1.062-1.074)$ \\
\hline Rural & $226,605(65.86)$ & $117,486(34.14)$ & $1.077(1.069-1.086)$ \\
\hline \multicolumn{4}{|l|}{ Charlson's comorbidity index } \\
\hline 0 & $616,812(65.75)$ & $321,322(34.25)$ & $1.050(1.041-1.058)$ \\
\hline 1 & $531,229(67.13)$ & $260,159(32.87)$ & $0.999(0.992-1.007)$ \\
\hline 2 & $338,054(67.95)$ & $159,469(32.05)$ & $0.962(0.955-0.970)$ \\
\hline$\geq 3$ & $461,701(66.71)$ & $230,416(33.29)$ & 1 (ref.) \\
\hline \multicolumn{4}{|l|}{ Comorbidity } \\
\hline Diabetes & $258,962(69.71)$ & $112,515(30.29)$ & $0.816(0.810-0.823)$ \\
\hline Dyslipidemia & $528,199(65.51)$ & $278,047(34.49)$ & $1.107(1.101-1.114)$ \\
\hline Congestive heart failure & $65,091(53.91)$ & $55,658(46.09)$ & $1.890(1.867-1.914)$ \\
\hline Ischemic heart failure & $257,083(64.02)$ & $144,464(35.98)$ & $1.132(1.123-1.140)$ \\
\hline Stroke & $87,896(64.83)$ & $47,673(35.17)$ & $1.141(1.128-1.155)$ \\
\hline Chronic kidney disease & $17,263(63.32)$ & $10,000(36.68)$ & $1.193(1.163-1.224)$ \\
\hline Cancer & $29,331(65.73)$ & $15,295(34.27)$ & $1.177(1.153-1.201)$ \\
\hline \multicolumn{4}{|l|}{ Provider type } \\
\hline General hospital & $641,364(69.37)$ & $283,228(30.63)$ & $0.767(0.763-0.772)$ \\
\hline Hospital & $216,869(65.53)$ & $114,059(34.47)$ & $0.964(0.956-0.971)$ \\
\hline Clinic & $1019,985(65.35)$ & $540,715(34.65)$ & 1 (ref.) \\
\hline Other & $69,578(67.59)$ & $33,364(32.41)$ & $0.900(0.888-0.913)$ \\
\hline \multicolumn{4}{|l|}{ Year of diagnosis } \\
\hline 2011 & $402,660(64.19)$ & $224,589(35.81)$ & 1 (ref.) \\
\hline 2012 & $395,541(66.15)$ & $202,371(33.85)$ & $0.915(0.909-0.922)$ \\
\hline 2013 & $395,450(68.36)$ & $183,029(31.64)$ & $0.824(0.818-0.831)$ \\
\hline 2014 & $364,782(67.74)$ & $173,686(32.26)$ & $0.843(0.837-0.850)$ \\
\hline 2015 & $389,363(67.47)$ & $187,691(32.53)$ & $0.850(0.843-0.856)$ \\
\hline
\end{tabular}

OR: odds ratio; CI: confidence interval.

ARBs were the most frequently prescribed antihypertensives in the nearby countries of Japan and China $[19,20]$. There may be several reasons for this changing prescription trend. The former JNC7 recommended diuretics as the first-line therapy $[6,21]$, but recent guidelines including regional Korean guidelines announced not only diuretics but also $A R B s$ and CCBs as the first-line treatment, and the corresponding drug utilization appears to have changed $[5,7,22,23]$. Moreover, although these guidelines included the diuretics as first-line drugs, in East Asian countries including Japan and China, the prescription rate of diuretics for hypertension is lower than that in the United States $[19,20,24]$. Frequent adverse events with the use of diuretics and associated low compliance may account for the low prescription rate $[12,19]$. Also, relatively low ACEi prescription presumably reflects the occurrence of frequent side effects in the Asian population such as dry cough [25]. The decreased use of ACEi might account for the increased use of 


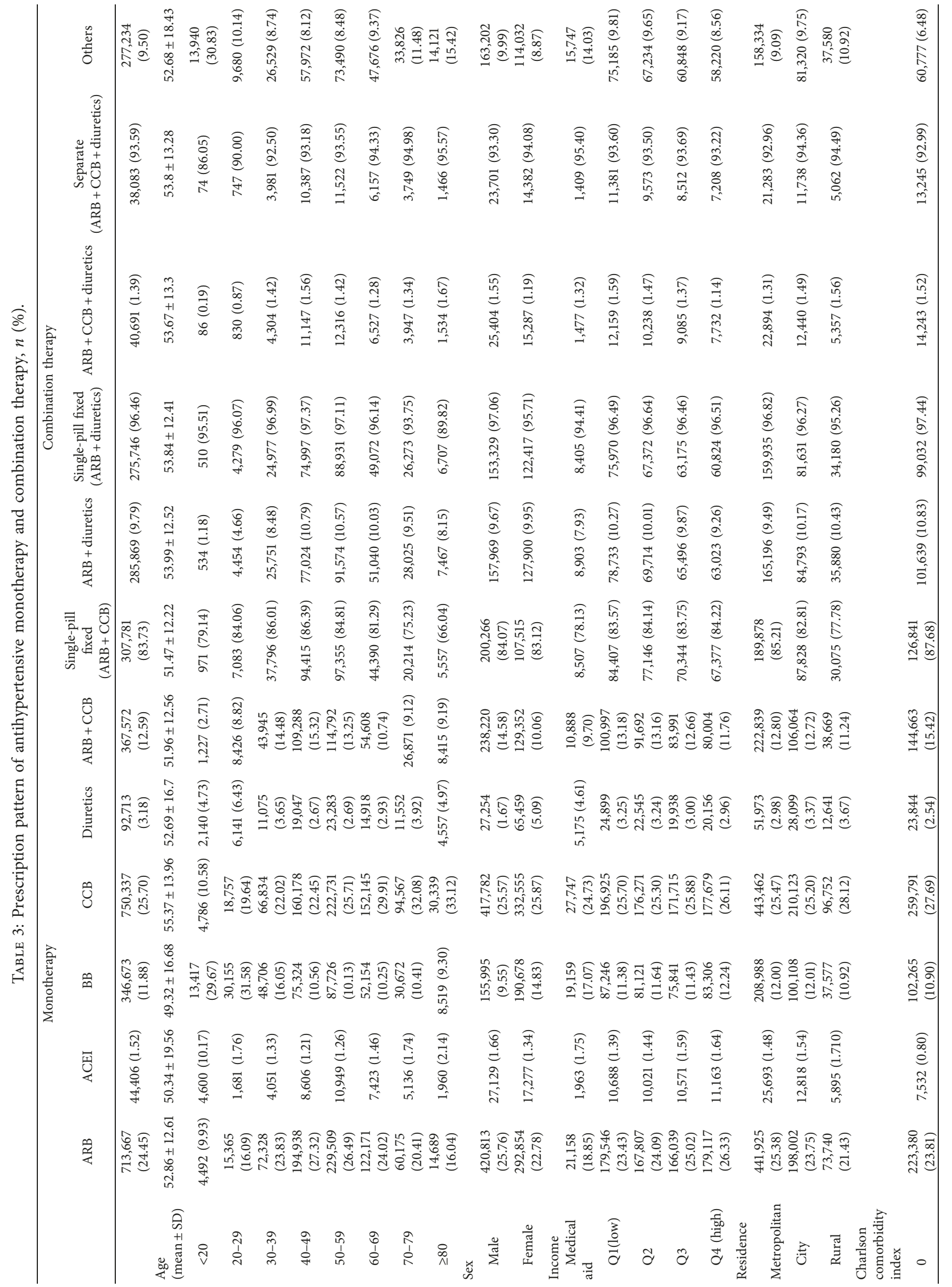




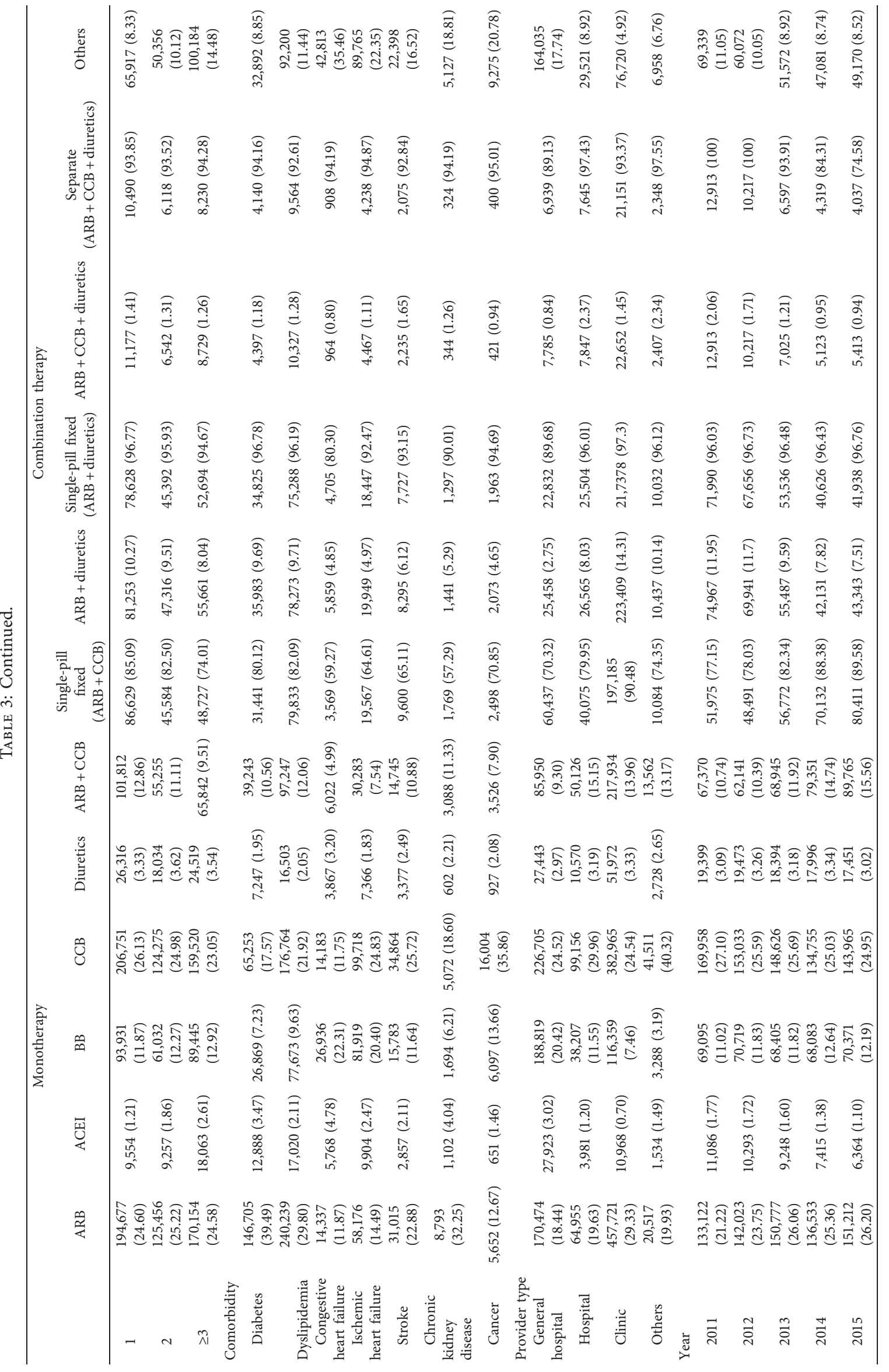


TABLe 4: Prescription pattern by the drug classes, either included in monotherapy or combination therapy, $n$ (\%).

\begin{tabular}{|c|c|c|c|c|c|}
\hline & $\mathrm{ARB}$ & ACEI & BETA & $\mathrm{CCB}$ & Diuretics \\
\hline Overall & $150,6561(51.61)$ & $90,784(3.11)$ & $539,372(18.48)$ & $1,314,597(45.03)$ & $496,634(17.01)$ \\
\hline Age $($ mean $\pm S D)$ & $53.01 \pm 12.74$ & $53.4 \pm 17.74$ & $51.43 \pm 16.17$ & $54.32 \pm 13.73$ & $54.07 \pm 13.81$ \\
\hline$<20$ & $6,928(15.32)$ & $5,564(12.30)$ & $15,015(33.20)$ & $7,479(16.54)$ & $3,092(6.84)$ \\
\hline $20-29$ & $31,458(32.94)$ & $2,561(2.68)$ & $35,064(36.72)$ & $32,134(33.65)$ & $13,038(13.65)$ \\
\hline $30-39$ & $156,422(51.54)$ & $7,447(2.45)$ & $66,968(22.06)$ & $130,704(43.06)$ & $48,151(15.86)$ \\
\hline $40-49$ & $415,900(58.29)$ & $17,660(2.48)$ & $118,228(16.57)$ & $315,763(44.25)$ & $124,418(17.44)$ \\
\hline $50-59$ & 476,338 (54.98) & $24,123(2.78)$ & $142,677(16.47)$ & $393,807(45.45)$ & $148,443(17.13)$ \\
\hline $60-69$ & $251,389(49.42)$ & $17,004(3.34)$ & $87,818(17.26)$ & $241,238(47.43)$ & $86,640(17.03)$ \\
\hline $70-79$ & $131,138(44.49)$ & 11,737 (3.98) & $55,469(18.82)$ & $145,191(49.26)$ & $54,624(18.53)$ \\
\hline$\geq 80$ & $36,988(40.38)$ & $4,688(5.12)$ & $18,133(19.80)$ & $48,281(52.71)$ & $18,228(19.90)$ \\
\hline \multicolumn{6}{|l|}{ Sex } \\
\hline Male & $904,778(55.38)$ & $60,192(3.68)$ & $275,763(16.88)$ & $775,090(47.44)$ & $251,286(15.38)$ \\
\hline Female & $601,783(46.82)$ & $30,592(2.38)$ & $263,609(20.51)$ & $539,507(41.97)$ & $245,348(19.09)$ \\
\hline \multicolumn{6}{|l|}{ Income } \\
\hline Medical aid & $46,315(41.27)$ & $3,943(3.51)$ & $27,160(24.20)$ & $46,751(41.66)$ & $19,055(16.98)$ \\
\hline Q1 (low) & $398,887(52.05)$ & $22,550(2.94)$ & $139,374(18.19)$ & $353,498(46.13)$ & $138,079(18.02)$ \\
\hline Q2 & $363,159(52.13)$ & $20,640(2.96)$ & $126,485(18.16)$ & $315,653(45.31)$ & $121,217(17.40)$ \\
\hline Q3 & $346,565(52.23)$ & $21,268(3.21)$ & $119,086(17.95)$ & $299,447(45.13)$ & $111,549(16.81)$ \\
\hline Q4 (high) & $351,635(51.68)$ & $22,383(3.29)$ & $127,267(18.7)$ & $299,248(43.98)$ & $106,734(15.69)$ \\
\hline \multicolumn{6}{|l|}{ Residence } \\
\hline Metropolitan & $909,496(52.23)$ & $53,311(3.06)$ & $320,725(18.42)$ & $778,121(44.69)$ & $282,095(16.20)$ \\
\hline City & $430,908(51.68)$ & $25,320(3.04)$ & $156,480(18.77)$ & $375,042(44.98)$ & $148,404(17.80)$ \\
\hline Rural & $166,157(48.29)$ & $12,153(3.53)$ & $62,167(18.07)$ & $161,434(46.92)$ & $66,135(19.22)$ \\
\hline \multicolumn{6}{|l|}{ Charlson comorbidity index } \\
\hline 0 & $502,861(53.60)$ & $12,245(1.31)$ & $137,490(14.66)$ & $456,193(48.63)$ & $163,131(17.39)$ \\
\hline 1 & $411,171(51.96)$ & $18,536(2.34)$ & $136,449(17.24)$ & $355,466(44.92)$ & $138,614(17.52)$ \\
\hline 2 & $252,680(50.79)$ & $19,096(3.84)$ & $97,293(19.56)$ & $212,683(42.75)$ & $85,095(17.10)$ \\
\hline$\geq 3$ & $339,849(49.10)$ & 40,907 (5.91) & $168,140(24.29)$ & $290,255(41.94)$ & $109,794(15.86)$ \\
\hline \multicolumn{6}{|l|}{ Comorbidity } \\
\hline Diabetes & $240,501(64.74)$ & $21,283(5.73)$ & $53,265(14.34)$ & $127,538(34.33)$ & $55,124(14.84)$ \\
\hline Dyslipidemia & $465,523(57.74)$ & $44,577(5.53)$ & $155,768(19.32)$ & $331,832(41.16)$ & $124,074(15.39)$ \\
\hline Congestive heart failure & $45,540(37.71)$ & $22,783(18.87)$ & $65,416(54.18)$ & $37,246(30.85)$ & $16,936(14.03)$ \\
\hline Ischemic heart failure & $148,348(36.94)$ & $40,612(10.11)$ & $161,776(40.29)$ & $178,876(44.55)$ & $44,121(10.99)$ \\
\hline Stroke & $67,621(49.88)$ & $6,568(4.84)$ & $34,636(25.55)$ & $66,925(49.37)$ & $19,360(14.28)$ \\
\hline Chronic kidney disease & $16,310(59.82)$ & $2,274(8.34)$ & $5,974(21.91)$ & $12,220(44.82)$ & $3,257(11.95)$ \\
\hline Cancer & $13,353(29.92)$ & $1,531(3.43)$ & $11,312(25.35)$ & $24,520(54.95)$ & $4,385(9.83)$ \\
\hline \multicolumn{6}{|l|}{ Provider type } \\
\hline General hospital & $350,910(37.95)$ & $67,357(7.29)$ & $316,088(34.19)$ & $406,074(43.92)$ & $81,064(8.77)$ \\
\hline Hospital & 161,403 (48.77) & $5,958(1.80)$ & $57,090(17.25)$ & $176,623(53.37)$ & $55,586(16.80)$ \\
\hline Clinic & $946,054(60.62)$ & $14,843(0.95)$ & $160,542(10.29)$ & $668,985(42.86)$ & $339,547(21.76)$ \\
\hline Others & $48,194(46.82)$ & $2,626(2.55)$ & $5,652(5.49)$ & $62,915(61.12)$ & $20,437(19.85)$ \\
\hline \multicolumn{6}{|l|}{ Year } \\
\hline 2011 & $312,074(49.75)$ & $21,853(3.48)$ & $115,374(18.39)$ & $291,626(46.49)$ & $132,317(21.09)$ \\
\hline 2012 & $306,353(51.24)$ & $20,206(3.38)$ & $112,748(18.86)$ & $257,977(43.15)$ & $117,653(19.68)$ \\
\hline 2013 & $300,076(51.87)$ & $18,703(3.23)$ & $104,476(18.06)$ & $252,941(43.73)$ & $94,175(16.28)$ \\
\hline 2014 & $280,036(52.01)$ & $15,514(2.88)$ & $101,408(18.83)$ & $245,769(45.64)$ & $76,045(14.12)$ \\
\hline 2015 & $308,022(53.38)$ & $14,508(2.51)$ & $105,366(18.26)$ & $266,284(46.15)$ & $76,444(13.25)$ \\
\hline
\end{tabular}

the alternative drug, the ARB. In addition, more favorable adherence and less frequent side effects might increase the use of ARB [12]. Lim et al. had already reported the increase in prescriptions for $\mathrm{ARBs}$ and a decrease for diuretics in Korea [15]. However, as the study was based on a single tertiary hospital, the results cannot be generalized to the overall population. Moreover, the study included patients who were already taking antihypertensives, so the proportion of combination therapy was higher than that of the present study (50.9\% vs. $33.3 \%)$. Although the prescription patterns of antihypertensives differ across countries $[11,19,20]$, the proportion of initial combination therapy of the present study seems to be low when compared to nearby countries [26]. Because intensive initial treatment for hypertension is recommended nowadays, an effort to increase the proportion of initial combination therapy may be warranted $[5,27]$. Considering patient compliance, a singlepill, fixed-dose combination is a good option [28].

In some groups, other classes rather than CCBs or ARBs were prescribed frequently. In those less than 20 years of age, the use of ACEi and BBs was more frequent. However, it is unclear why ACEi and BBs were used more frequently than in other age groups. Certain underlying conditions such as diabetes, proteinuria, or migraines as well as physician's 


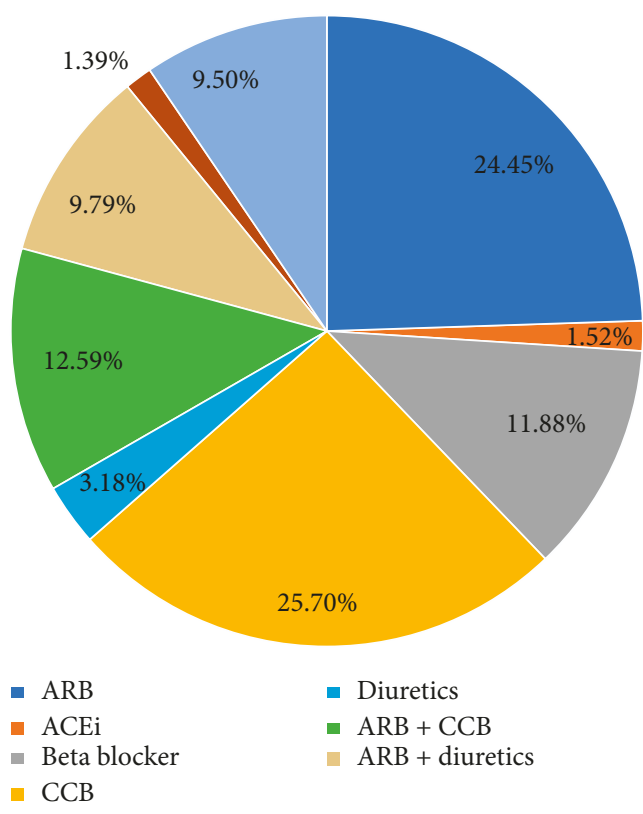

(a)

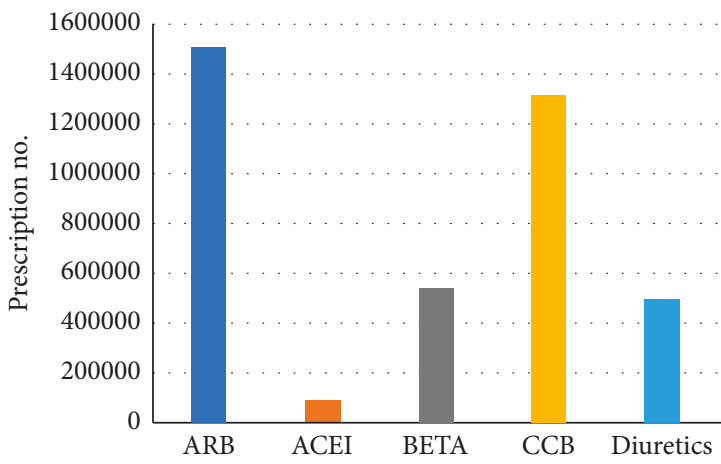

(c)

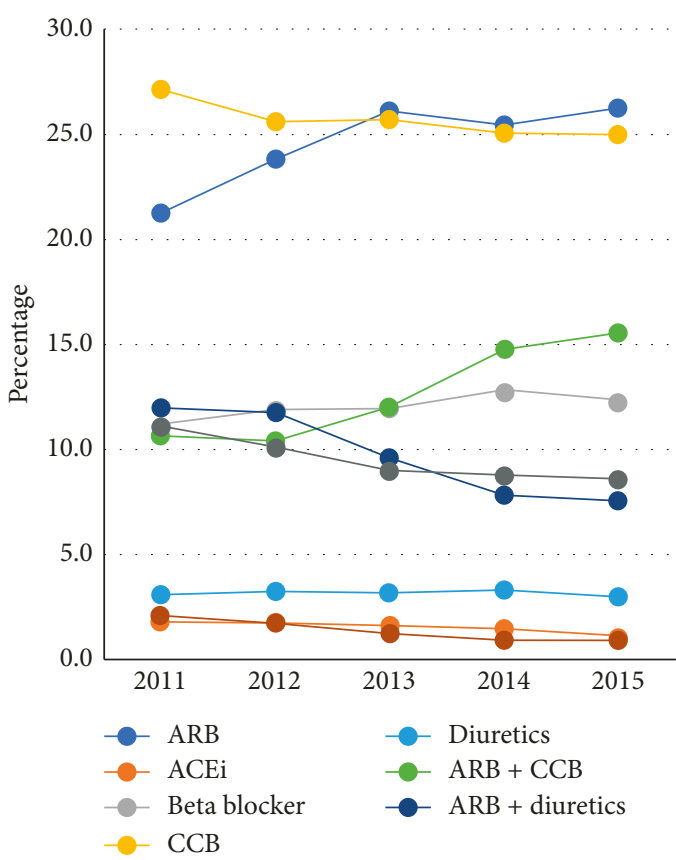

(b)

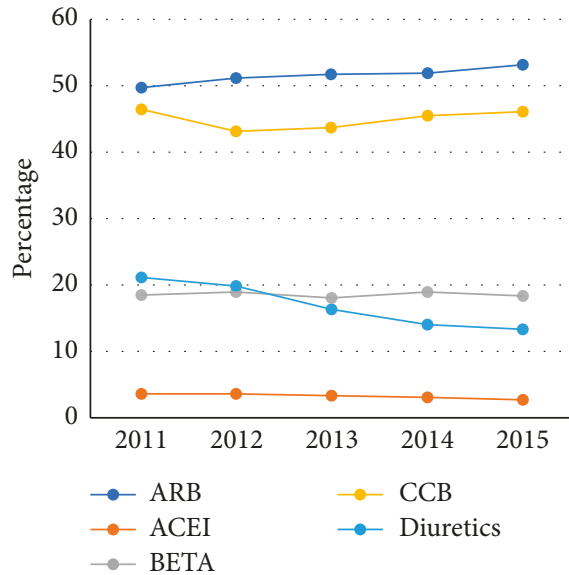

(d)

Figure 1: Prescription pattern and trend of the antihypertensive drugs. (a) Prescription pattern including combination therapy, (b) trend including combination therapy, (c) prescription number by drug classes, and (d) trend by drug classes.

preference considering adverse drug effects may affect the prescription pattern in children and adolescents $[29,30]$. Although several guidelines excluded beta-blocker as a firstline antihypertensive drug $[5,7]$, the prescription rate of the drug was relatively stable during the study period. It may be due to the presence of comorbid conditions such as ischemic heart disease and arrhythmia, in which the use of betablockers is inevitable. However, overall beta-blocker use was found to be relatively low. Unlike the JNC 7 guideline, the JNC 8 guideline did not suggest compelling indications of each antihypertensive drug classes. However, there are several clinical situations which make a specific antihypertensive class preferred to others. For example, JNC 7 and domestic hypertension guidelines in Korea recommend beta-blocker as an initial choice for hypertension in congestive heart failure patients [16]. Therefore, unlike other conditions, beta-blocker may be most frequently prescribed for congestive heart failure patients in the present study, suggesting that clinical guidelines are reflected in prescription of Korean physicians.

On the contrary, we also observed some gaps between the guidelines and clinical practice. According to the JNC 8 guideline, the use of ARB or ACEi was recommended especially in those with chronic kidney diseases [7]. However, the prescription rate of ARB or ACEi in the chronic kidney disease patients was not optimal and was only $68.2 \%$. Even though there can be rational reasons not to use these drugs, a substantial portion of chronic kidney disease patients may lose the chance to prevent or delay disease progression. Doctors often prescribe drugs according to prescription 
patterns based on their own experience rather than scientific evidences [11]. Further studies are needed to assess and decrease these gaps of prescription pattern.

Overall, the prescription rate of combination therapy was slightly decreasing throughout the study period. In JNC 8 guideline, patients with stage 2 hypertension $(\mathrm{BP}>160$ / 100) are recommended to receive combination therapy from the first prescription [7]. In Korea, the national health screening program is offered biennially and more hypertension is diagnosed at an earlier stage due to increasing participation rates [31]. However, considering the relatively low proportion of patients achieving the target blood pressure level (less than 70\%) [32], more aggressive treatment for hypertensive patients using combination therapy is warranted.

For initial combination therapy, most patients received single-pill fixed-dose combination drugs and this was an increasing trend. The use of single-pill fixed-dose combination is advantageous in terms of the price of a single-pill combination and the advantage of drug adherence and blood pressure control $[6,7,11,33]$, suggesting a trend toward the favorable prescription pattern.

There are several limitations to be considered in this study. First, there is possibility of false claims of hypertension for reimbursement purpose. For example, betablockers are often effective in chronic headache but not reimbursed in Korea for that purpose. So, the physician might have entered the hypertension disease code for making the drug covered by the NHI. Second, we do not have detailed clinical information for each prescription and could not determine the appropriateness of drug choice in individual basis.

\section{Conclusions}

We identified overall prescription patterns of antihypertensives for treatment-naive patients in South Korea. The findings can lead to a rational, evidence-based, and costeffective improvement of prescription patterns in newly diagnosed hypertensive patients.

\section{Data Availability}

The data are available at the online public repository of the National Health Insurance Sharing Service (https://nhiss. nhis.or.kr/bd/ay/bdaya001iv.do). As the data are owned by the National Health Insurance, institutional approval must precede before providing the dataset.

\section{Conflicts of Interest}

Kim Y.-H., Jeon S.-A., and Kwon Y.-C. are Pfizer employees. However, they made no influence on this work in relation with the company or its products. Other authors have no potential conflicts of interest to disclose.

\section{Acknowledgments}

The authors would like to thank Ms. Tanaya Bharatan, Pfizer, for her editorial support for this manuscript. This study was sponsored by Pfizer Pharmaceutical Korea Ltd (Grant no. X9001150).

\section{References}

[1] M. Ezzati, A. D. Lopez, A. Rodgers, S. Vander Hoorn, C. J. Murray, and G. Comparative Risk Assessment Collaborating, "Selected major risk factors and global and regional burden of disease," The Lancet, vol. 360, no. 9343, pp. 1347-1360, 2002.

[2] P. M. Kearney, M. Whelton, K. Reynolds, P. Muntner, P. K. Whelton, and J. He, "Global burden of hypertension: analysis of worldwide data," The Lancet, vol. 365, no. 9455, pp. 217-223, 2005.

[3] D. E. Bloom, S. Chen, M. Kuhn, M. McGovern, L. T. Oxley, and K. Prettner, "The economic burden of chronic diseases: estimates and projections for China, Japan, and South Korea," Hohenheim Discussion Papers in Business, Economics and Social Sciences, University of Hohenheim, Stuttgart, Germany, 2017.

[4] P. M. Kearney, M. Whelton, K. Reynolds, P. K. Whelton, and J. He, "Worldwide prevalence of hypertension," Journal of Hypertension, vol. 22, no. 1, pp. 11-19, 2004.

[5] P. K. Whelton, R. M. Carey, W. S Aronow et al., "2017 ACC/ AHA/AAPA/ABC/ACPM/AGS/APhA/ASH/ASPC/NMA/ PCNA guideline for the prevention, detection, evaluation, and management of high blood pressure in adults: a report of the American College of Cardiology/American heart association task force on clinical practice guidelines," Journal of the American College of Cardiology, vol. 71, no. 19, pp. e127-e248, 2018.

[6] A. V. Chobanian, G. L. Bakris, H. R. Black et al., "Seventh report of the joint national Committee on prevention, detection, evaluation, and treatment of high blood pressure," Hypertension, vol. 42, no. 6, pp. 1206-1252, 2003.

[7] P. A. James, S. Oparil, B. L. Carter et al., "2014 evidence-based guideline for the management of high blood pressure in adults," JAMA, vol. 311, no. 5, pp. 507-520, 2014.

[8] M. C. S. Wong, J. Y. Jiang, and S. M. Griffiths, "Factors associated with compliance, discontinuation and switching of calcium channel blockers in 20,156 Chinese patients," American Journal of Hypertension, vol. 22, no. 8, pp. 904-910, 2009.

[9] M. C. S. Wong, W. W. S. Tam, C. S. K. Cheung et al., "Initial antihypertensive prescription and switching: a 5 year cohort study from 250,851 patients," PLoS One, vol. 8, no. 1, Article ID e53625, 2013.

[10] K. Kretzer, D. T. Juarez, and J. Davis, "Initial antihypertensive prescriptions, switching patterns and adherence among insured patients in Hawai'i," Hawaii Medical Journal, vol. 67, no. 4, pp. 96-99, 2008.

[11] N. Jarari, N. Rao, J. R. Peela et al., "A review on prescribing patterns of antihypertensive drugs," Clinical Hypertension, vol. 22, p. 7, 2015.

[12] Y.-M. Ah, J.-Y. Lee, Y.-J. Choi et al., "Persistence with antihypertensive medications in uncomplicated treatment-naïve patients: effects of initial therapeutic classes," Journal of Korean Medical Science, vol. 30, no. 12, pp. 1800-1806, 2015.

[13] M. C. S. Wong, J. Y. Jiang, A. T. Lam, H. Fung, S. Griffiths, and S. W. Mercer, "Patterns of antihypertensive prescribing, discontinuation and switching among a Hong Kong Chinese population from over one million prescriptions," Journal of Human Hypertension, vol. 22, no. 10, pp. 714-716, 2008. 
[14] G. Mazzaglia, L. G. Mantovani, M. C. Sturkenboom et al., "Patterns of persistence with antihypertensive medications in newly diagnosed hypertensive patients in Italy: a retrospective cohort study in primary care," Journal of Hypertension, vol. 23, no. 11, pp. 2093-2100, 2005.

[15] B. Lim, E. Kang, S.-h. Yoo, and S. Sunwoo, "Utilization pattern of anti-hypertensive medications according to comorbidities in a hospital-based family medicine practice," Korean Journal of Family Practice, vol. 7, no. 4, pp. 563-568, 2017.

[16] Y.-N. Sung, S.-M. Jang, D.-H. Lim, S.-Y. Shin, H.-J. Song, and S.-H. Lee, "Prescribing patterns of antihypertensive drugs by outpatients with hypertension in 2007," Korean Journal of Clinical Pharmacy, vol. 19, 2009.

[17] D. W. Shin, B. Cho, and E. Guallar, "Korean national health insurance Database," JAMA Internal Medicine, vol. 176, no. 1, p. 138, 2016.

[18] J. Lee, J. S. Lee, S. H. Park, S. A. Shin, and K. Kim, “Cohort profile: the national health insurance service-national sample cohort (NHIS-NSC), South Korea," International Journal of Epidemiology, vol. 46, p. e15, 2017.

[19] T. Kohro, T. Yamazaki, H. Sato, K. Ohe, and R. Nagai, “The impact of a change in hypertension management guidelines on diuretic use in Japan: trends in antihypertensive drug prescriptions from 2005 to 2011," Hypertension Research, vol. 36, no. 6, pp. 559-563, 2013.

[20] H. Xu, Y. He, L. Xu, X. Yan, and H. Dai, “Trends and patterns of five antihypertensive drug classes between 2007 and 2012 in China using hospital prescription data," International Journal of Clinical Pharmacology and Therapeutics, vol. 53, no. 6, pp. 430-437, 2015.

[21] C. Lenfant, A. V. Chobanian, D. W. Jones, and E. J. Roccella, "Seventh report of the joint national Committee on the prevention, detection, evaluation, and treatment of high blood pressure (JNC 7)," Hypertension, vol. 41, no. 6, pp. 1178-1179, 2003.

[22] Korean Society of Hypertension, 2013 Korean Society of Hypertension Guidelines for the Management of Hypertension, Korean Society of Hypertension, Seoul, South Korea, 2013.

[23] Korean Society of Hypertension, 2018 Korean Society of Hypertension Guidelines for the Management of Hypertension, Korean Society of Hypertension, Seoul, South Korea, 2018.

[24] S. J. Shah and R. S. Stafford, "Current trends of hypertension treatment in the United States," American Journal of Hypertension, vol. 30, no. 10, pp. 1008-1014, 2017.

[25] S. E. McDowell, J. J. Coleman, and R. E. Ferner, "Systematic review and meta-analysis of ethnic differences in risks of adverse reactions to drugs used in cardiovascular medicine," BMJ, vol. 332, no. 7551, pp. 1177-1181, 2006.

[26] P. H. Liu and J. D. Wang, "Antihypertensive medication prescription patterns and time trends for newly-diagnosed uncomplicated hypertension patients in Taiwan," BMC Health Services Research, vol. 8, p. 133, 2008.

[27] T. M. MacDonald, B. Williams, D. J Webb et al., "British hypertension society programme of $\mathrm{P}$, treatment of hypertension with algorithm-based T. Combination therapy is superior to sequential monotherapy for the initial treatment of hypertension: a double-blind randomized controlled trial," Journal of the American Heart Association, vol. 6, no. 11, 2017.

[28] S. Bangalore, G. Kamalakkannan, S. Parkar, and F. H. Messerli, "Fixed-dose combinations improve medication compliance: a meta-analysis," The American Journal of Medicine, vol. 120, no. 8, pp. 713-719, 2007.
[29] G. B. Luma and R. T. Spiotta, "Hypertension in children and adolescents," American Family Physician, vol. 73, pp. 15581568, 2006.

[30] E. H. Lee, H. E. Yim, G. Y. Jang et al., "Evaluating and managing hypertension in children: a survey of Korean cardiologists and nephrologists," Korean Journal of Pediatrics, vol. 51, no. 9, pp. 992-997, 2008.

[31] H. Lee, J. Cho, D. W. Shin et al., "Association of cardiovascular health screening with mortality, clinical outcomes, and health care cost: a nationwide cohort study," Preventive Medicine, vol. 70, pp. 19-25, 2015.

[32] KCDC, Korean Health Statistics 2013: Korea National Health and Nutrition Examination Survey (KNHANES VI-1), 2014.

[33] Q. Gu, V. L. Burt, C. F. Dillon, and S. Yoon, "Trends in antihypertensive medication use and blood pressure control among United States adults with hypertension," Circulation, vol. 126, no. 17, pp. 2105-2114, 2012. 


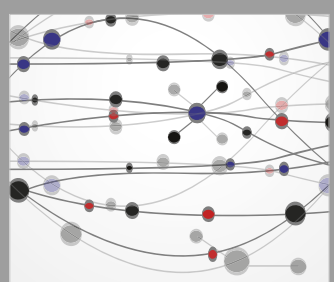

The Scientific World Journal
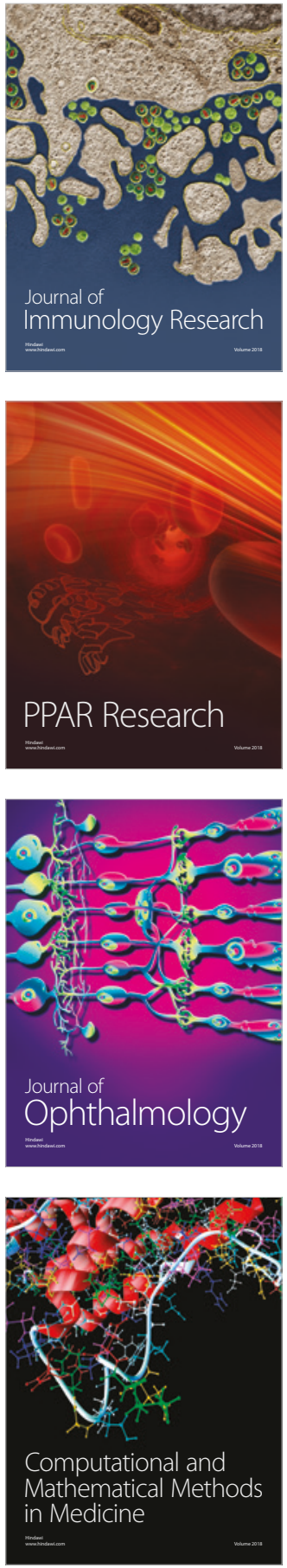

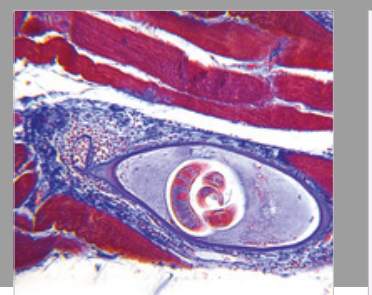

Gastroenterology Research and Practice

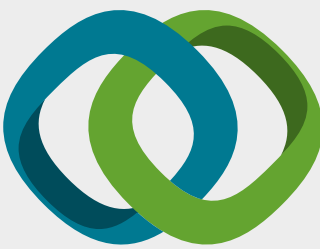

\section{Hindawi}

Submit your manuscripts at

www.hindawi.com
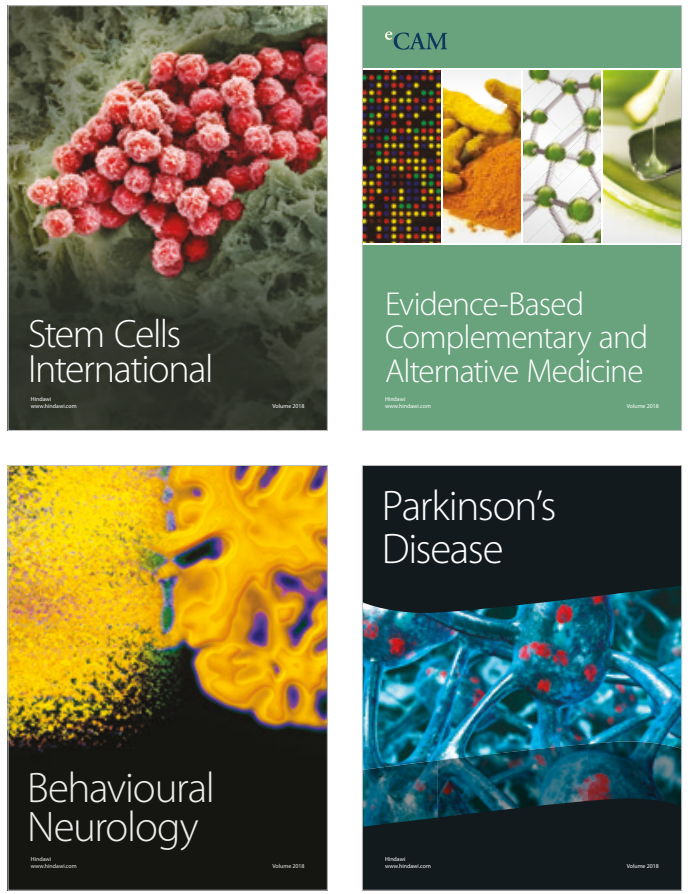



ournal of

Diabetes Research

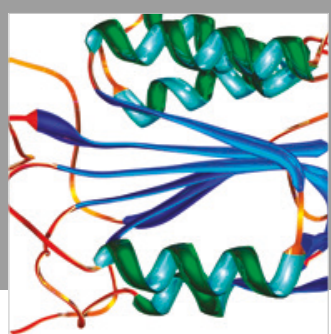

Disease Markers
